\title{
CAROTID PLAQUE CLASSIFICATION USING CONTOURLET FEATURES AND SUPPORT VECTOR MACHINES
}

\author{
${ }^{1} \mathrm{M}$. Thangavel, ${ }^{2} \mathrm{M}$. Chandrasekaran and ${ }^{3} \mathrm{M}$. Madheswaran \\ ${ }^{1}$ Department of ECE, Knowledge Institute of Technology, Salem, India \\ ${ }^{2}$ Department of ECE, Government College of Engineering, Bargur, India \\ ${ }^{3}$ Department of ECE, Mahendra Engineering College, Namakkal, India
}

Received 2014-02-03; Revised 2014-02-14; Accepted 2014-04-15

\begin{abstract}
The aim of this study is to propose a suitable and reliable system for better diagnosis and treatment of carotid diseases. In this study, Computer Aided Diagnosis (CAD) system has been proposed for classifying carotid artery plaques using Contourlet features. Carotid images have been acquired for 124 subjects with symptoms (Amaurosis Fugax, Stroke or Transient Ischemic Attack) and 133 subjects with no symptoms in the recent past. Images were normalized and plaque regions have been manually segmented by experts and these Region Of Interests (ROI) have been used for further processing. 4 level Contourlet transform has been applied to all ROIs and subimages were produced at different scales and orientations. Energy, Entropy, Mean and Standard deviation features were extracted from all the subimages. The feature selection has been done to select significant features and to ignore insignificant ones. Support Vector Machine classifier (SVM) and Adaboost classifier have been applied to the selected features and plaques were classified as symptomatic or asymptomatic plaques. The contourlet features with Support vector machine classifier produced classification accuracy of $85.6 \%$ compared to $81.3 \%$ accuracy in Adaboost classifier. The classification results were compared with curvelet transform features and wavelet packet features. The contourlet with SVM classifier yielded better performance compared to curvelet and wavelet packet.
\end{abstract}

Keywords: Ultrasound, Contourlet, Plaque, Segmentation, Classification, Symptomatic, Asymptomatic

\section{INTRODUCTION}

Stroke has been one of the leading causes of death in the world and about 6.2 million people die each year around the globe due to stroke. Approximately 15 million people worldwide have a stroke each year (WHO 2013). 1 in 6 people around the world will have a stroke in their lifetime. Two-thirds of stroke deaths occur in less developed countries. A stroke or brain attack is similar to a heart attack and is mostly caused by a blockage of a blood vessel to the brain. In some cases, it can also be caused by an area of bleeding into the brain, referred to as a hemorrhagic stroke. Transient Ischemic Attack (TIA) is a set of symptoms that occur as a result of a temporary lack of blood supply to the brain. These symptoms last only a few minutes and then completely resolve. Strokes can affect people in different ways depending on which part of the brain has been affected. An ischemic stroke may be caused by different kinds of diseases. The main problem for the stroke is narrowing of arteries either in neck or in head. If the arteries become too narrow, blood clots are formed due to collection of blood cells. These blood clots can block the artery in the place of formation or it can move and get trapped in arteries closer to brain.

Carotid atherosclerosis is the reason for most of the strokes. Atherosclerosis is a condition in which carotid artery becomes narrow due to accumulation of calcium and fatty substances. These excessive building up of these substances form plaques in the lumen area of the artery. Plaques can be of two types, namely stable plaque and unstable or vulnerable plaque. Stable plaques are asymptomatic and may exist for long period of time without any symptoms. Unstable plaques are dangerous

Corresponding Author: M. Thangavel, Department of ECE, Knowledge Institute of Technology, Salem, India 
and exhibit symptoms such as Transient ischemia attack, Amaurosis Fugax (AF) prior to stroke. The ultrasound imaging play vital role in diagnosing the carotid atherosclerosis because of the many advantages.

Plaque tissue has been characterized and classified into symptomatic or asymptomatic using grey scale features and degree of stenos is. Significant features were selected based on divergence value and correlation. SVM classifier was used for classification of plaques. A novel Plaque risk index has been proposed using the extracted features to objectively classify the plaques and to monitor the changes in plaque over period (Acharya et al., 2013a).

An automated computer aided diagnosis system have been developed for plaque classification. Statistical and structural approaches were used for extracting the features. Classification has been performed using Adaboost and Support Vector Machines classifiers. To discriminate symptomatic and asymptomatic plaques and to help the physicians for quantifying the severity of plaques, an symptomatic asymptomatic carotid index has been used (Acharya et al., 2012a). Carotid atherosclerosis has been analyzed and assessed using three multiscale transforms (Tsiaparas et al., 2012). Texture features were extracted from detail subimages obtained after applying different transforms. Features were selected based on the separability value and correlation. To overcome the availability of limited samples, bootstrapping technique has been used to resample the selected features. SVM has been used for classification. Curvelet transform has yielded high classification accuracy compared to other multiscale transforms used. Discrete wavelet features along with higher order spectra and texture features have been used for testing the performance of SVM classifier with different kernels (Acharya et al., 2011). Usage of higher order spectra enhanced the classification accuracy.

The efficiency of different wavelet transforms with respect to plaque classification has been tested using the multi resolution features. Nonlinear correlation coefficient has been used to select the feature based on the threshold value. SVM and probabilistic neural network classifiers were used for classifying carotid plaques (Tsiaparas et al., 2011). The Gabor transform, statistical and fractal features were also extracted for comparing the performance. Wavelet packet with coiflet 1 basis function exhibited superior performance with SVM classifier. Multilevel binary morphological model and gray scale morphological model were used to obtain pattern spectra features and classification have been done using probabilistic neural network and SVM classifiers (Kyriacou et al., 2009). Multilevel binary morphological model outperformed gray scale model in classification efficiency. Morphological measurements agreed with clinical measurements. Very high classification accuracy has been obtained by Mougiakakou et al. (2007) using a hybrid method. First order statistical features and laws' texture energy were extracted from the images with plaques. By using Analysis Of Variance (ANOVA), dimension of the extracted features was reduced. Neural network based on genetic algorithm was used for classification. The higher classification accuracy was due to the usage of hybrid classification system. A modular neural network based classification was used for ten different features. The performance of Self Organizing Map (SOM) and K Nearest Neighbor (KNN) were tested. The quality of ultrasound images affect the quality of extracted features and improves the classification performance (Christodoulou et al., 2003).

Do and Vitterly (2005) proposed the contourlet transform for representing images and it was shown that contourlet transform represented contours optimally compared to wavelet transform. The contourlet transform has produced a gain of $1.46 \mathrm{~dB}$ in peak signal to noise ratio compared to wavelet. The contourlet transform (Katsigiannis et al., 2010) has been applied to thyroid ultrasound images to classify textures using support vector machine classifier with classification accuracy of 95\% for hypoechoic dataset and $92 \%$ for isoehoic dataset. Long and Younan (2006) applied Contourlet transform for sets of Brodatz textures and classified the textures with high accuracy. Contourlet spectral histogram outperformed Wavelet energy histogram and wavelet spectral histogram with respect to Classification accuracy. Moayedi et al. (2010) developed an automatic mass classification system for classifying mammogram images using contourlet features and Support vector machines. A high classification accuracy was obtained for successive enhance learning weighted SVM. Wavelet and contourlet features were extracted and they were combined for classification of images selected from Brodatz datasets using SVM classifier. The accuracy of classification increased when features were combined (Li and Shawe-Taylor 2004). In this study a CAD has been proposed to classify carotid plaques using contourlet transform features. Figure 1 shows the proposed CAD system for classification of plaques. 


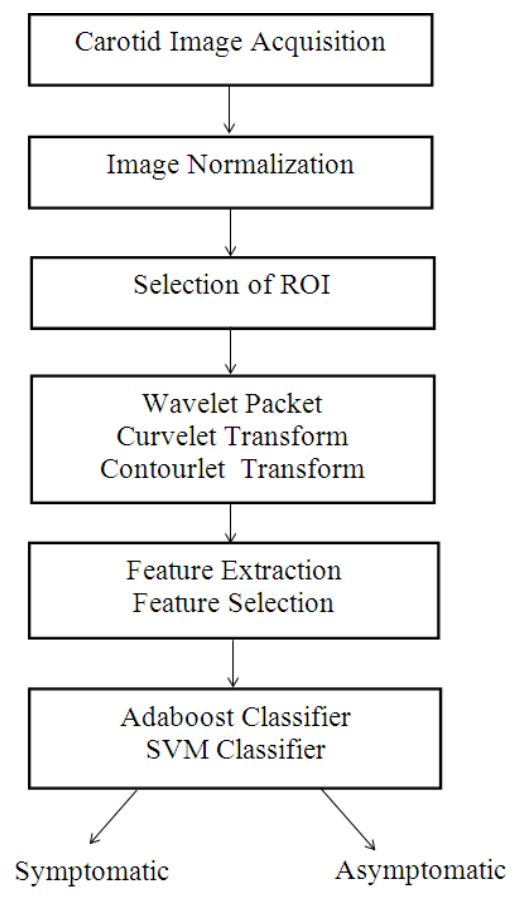

Fig. 1. Block diagram of proposed computer

\section{MATERIALS AND METHODS}

\subsection{Algorithm}

The algorithm for the proposed CAD system is as follows:

Step 1: Image acquisition

Step 2: Image normalization

Step 3: Selection of ROI

Step 4: Applying multiscale transform

Step 5: Features extraction and selection

Step 7: Classification of carotid plaques

\subsection{Contourlet Transform}

The contourlet transform was developed by Do and Vitterly (2002) for efficient representation of images that are anisotropic in nature. The wavelet transform can efficiently represent images that have isotropic structures. For images with anisotropic structures, representation of images using wavelet is not optimal. The contourlet transform can effectively represent smooth contours and geometric structures. Like wavelet transform, contourlet transform represents the image in different scales and directions. The contourlet transform is implemented using double filter bank structures. First, Laplacian pyramid (Burt and Adelson, 1983) is used to capture point discontinuities in the images. Then Directional filter bank is used (Bamberger and Smith, 1992) to link point discontinuities to get linear structure.

The implementation of contourlet transform is shown in Figure 2. The input image is applied to Laplacian pyramid to generate down sampled low pass version of input image and band pass image. The band pass image is filtered by directional filter bank to get directional information. The low pass image is further decomposed to produce band pass image and down sampled low pass image and this procedure is repeated for many levels.

\subsection{Image Acquisition and Normalization}

For our study, a total of 257 (124 symptomatic and 133 asymptomatic) carotid B mode longitudinal images were acquired. To overcome the irregularities induced in the images due to different gain settings, different light conditions and also variations due to different operators, the images were normalized to distribute the histogram of the images evenly. The region of interest has been selected manually using the experts and were separated from the rest of the image portion and stored in the computer for further processing.

\subsection{Feature Extraction and Selection}

For the selected ROI, first Laplacian pyramid has been used to obtain multiscale decomposition. Using the directional filter bank, each band pass image is decomposed into subimages in different directions. The number of direction chosen were $4,4,8$ and 16 from finest scale to coarsest scale. The total number of detailed sub images generated were 32. For each sub image, mean, standard viation, energy and entropy features were extracted. The equation for features extracted are given below Equation 1 to 4:

$$
\begin{aligned}
& \operatorname{Energy~}_{j k}=\frac{1}{M \cdot N} \sum_{x=1}^{N} \sum_{y=1}^{M}\left[I_{j k}(x, y)\right]^{2} \\
& \text { Mean } \mu_{j k}=\frac{1}{M \cdot N} \sum_{x=1}^{N} \sum_{y=1}^{M} I_{j k}(x, y) \\
& \text { Standard deviation } \sigma_{j k}=\sqrt{\frac{1}{M \cdot N} \sum_{x=1}^{N} \sum_{y=1}^{M}\left(I_{j k}(x, y)-\mu_{j k}\right)^{2}} \\
& \text { Entropy } e_{j k}=-\frac{1}{N \cdot M} \sum_{x=1}^{N} \sum_{y=1}^{M} \mu_{j k}^{2} \log \left(\mu_{j k}\right)^{2}
\end{aligned}
$$




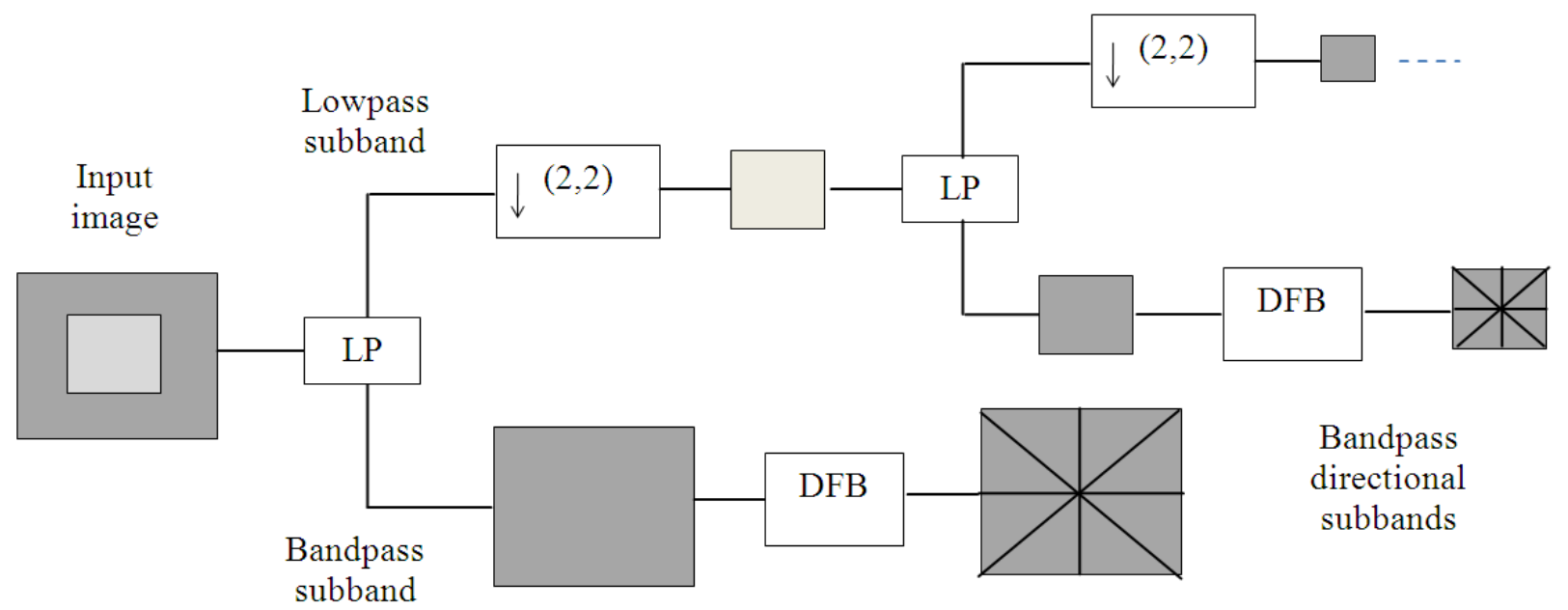

Fig. 2. Implementation of Contourlet transform using filter banks

where, $I_{j k}$ is the sub band image obtained at $j^{\text {th }}$ level and $\mathrm{k}^{\text {th }}$ direction.

A total of 128 features have been obtained. Here low pass image has not taken into consideration for feature extraction as the most of the texture information were filtered out due to repeated low pass filtering. As the large number of features were generated in all the transforms considered for features extraction, the feature selection has been used to select the significant features and to ignore the insignificant features. The feature selection not only improves the performance of classification algorithms but also reduces the computation complexity. Feature vectors for each subband were formed by combining the all the feature extracted. Then the mean for each vectors was calculated. The standard deviation between the different means were also calculated. A hard threshold was applied to standard deviation and 73 significant features were retained.

\section{RESULTS}

For this study, 257 carotid ultrasound images [124 symptomatic and 133 asymptomatic] have been used. Figure 3a and $\mathbf{b}$ respectively show the symptomatic and asymptomatic plaque regions segmented from ultrasound images. Contourlet transform has been applied to decompose the region of interest into subbands. Figure $4 \mathbf{a}$ and $\mathbf{b}$ show the decomposed images using Contourlet transform for symptomatic and asymptomatic plaques. For each subimage, mean, standard deviation, energy and entropy features has been extracted and significant features have been retained and insignificant features have been discarded. SVM and Adaboost classifiers have been used for classification of symptomatic and asymptomatic images. The curvelet transform and wavelet packet have also been applied to all the selected Region of interests and classification was done using SVM and adaboost classifiers from the selected features. Table 1 shows the performance of SVM classifier for wavelet packet, curvelet and contourlet transform features. The classification accuracy for SVM classifier with wavelet packet, curvelet and contourlet transform features are 78.6, 81.3 and $85.6 \%$ respectively. The performance of adaboost classifier for wavelet packet, curvelet and contourlet features is given in Table 2 . The adaboost classifier yielded the classification accuracy of 75.1, 79.4 and $81.3 \%$ respectively for wavelet packet, curvelet and contourlet transform features. For both SVM and adaboost classifier the classification accuracy is the highest for contourlet transform features compared to accuracy of wavelet packet and curvelet transform. Figure 5a and b show the Receiver Operating Characteristics (ROC) curves of SVM and Adaboost classifiers for the three transform features considered. 
Table 1. Performance of SVM classifier

\begin{tabular}{|c|c|c|c|c|c|c|c|c|c|c|}
\hline & $\mathrm{TP}$ & $\mathrm{TN}$ & FP & $\mathrm{FN}$ & Accuracy $\%$ & Sensitivity\% & Specificity\% & PPV\% & NPV\% & AUC \\
\hline Wavelet packet & 99 & 103 & 30 & 25 & 78.6 & 79.8 & 77.4 & 76.7 & 80.5 & 0.75 \\
\hline Curvelet & 101 & 108 & 25 & 23 & 81.3 & 81.5 & 81.2 & 80.2 & 82.4 & 0.82 \\
\hline Contourlet & 108 & 112 & 21 & 16 & 85.6 & 87.1 & 84.2 & 83.7 & 87.5 & 0.86 \\
\hline
\end{tabular}

Table 2. Performance of Adaboost classifier

\begin{tabular}{lllllllllll}
\hline & TP & TN & FP & FN & Accuracy & \%Sensitivity\% & Specificity\% & PPV\% & NPV & AUC \\
\hline Wavelet packet & 95 & 98 & 35 & 29 & 75.1 & 76.6 & 73.7 & 73.1 & 77.2 & 0.73 \\
Curvelet & 99 & 105 & 28 & 25 & 79.4 & 79.8 & 78.9 & 78.0 & 80.8 & 0.81 \\
Contourlet & 97 & 112 & 21 & 27 & 81.3 & 78.2 & 84.2 & 82.2 & 80.6 & 0.82 \\
\hline
\end{tabular}

TP-True Positive; TN-True Negative; FP-False Positive; FN-False Negative; PPV-Positive Predictive Value

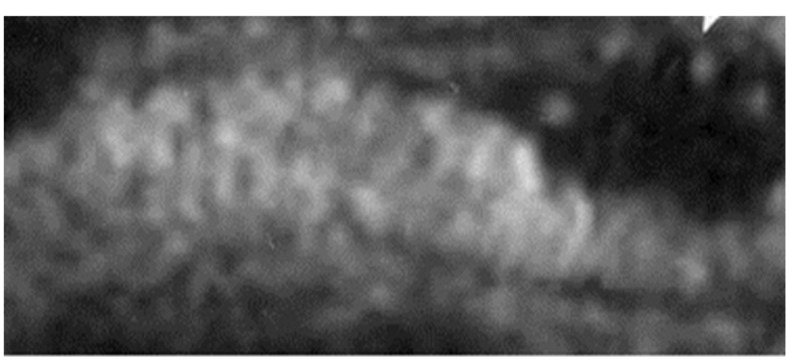

(a)

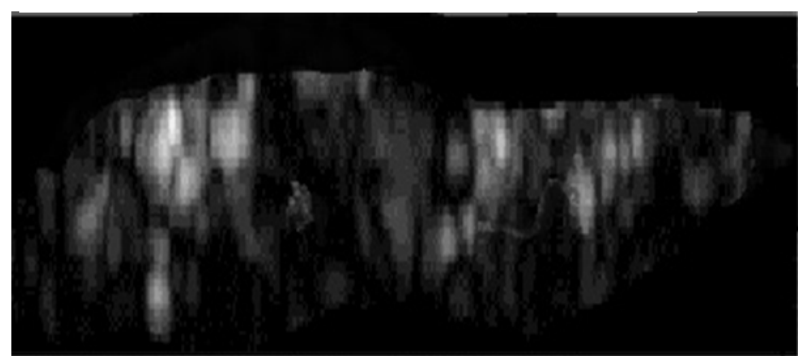

(b)

Fig. 3. (a) Symptomatic plaque (b) asymptomatic plaque

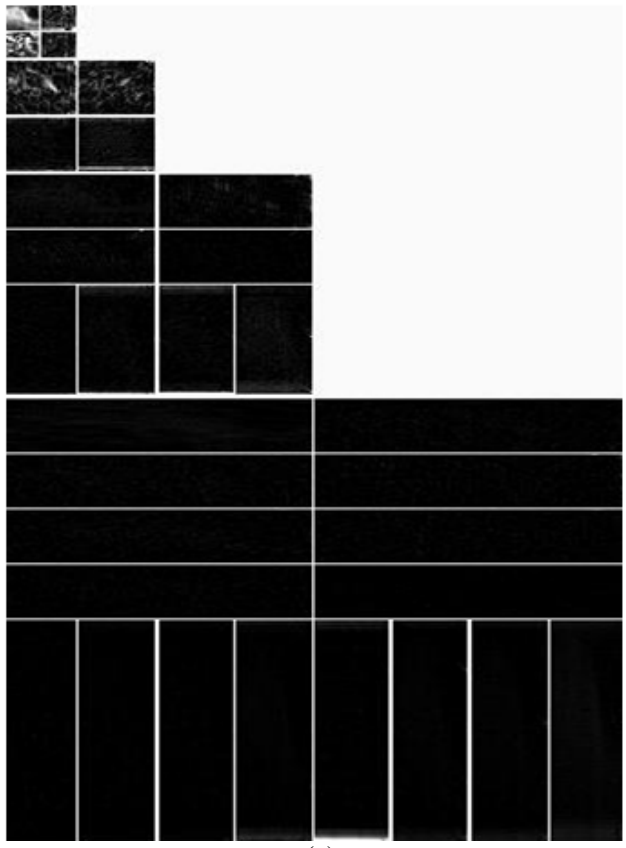

(a)

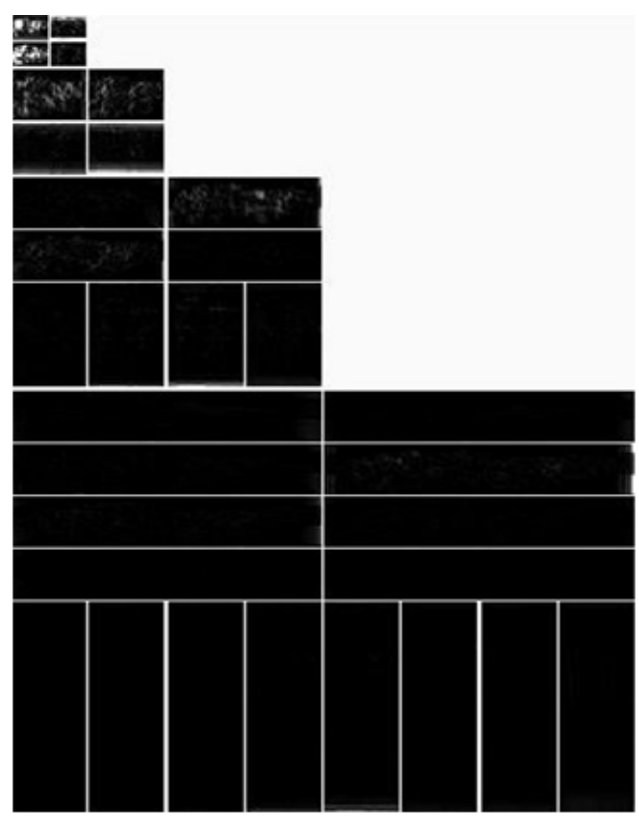

(b)

Fig. 4. (a) Contourlet coefficients of symptomatic plaque (b) contourlet coefficients of asymptomatic plaque 


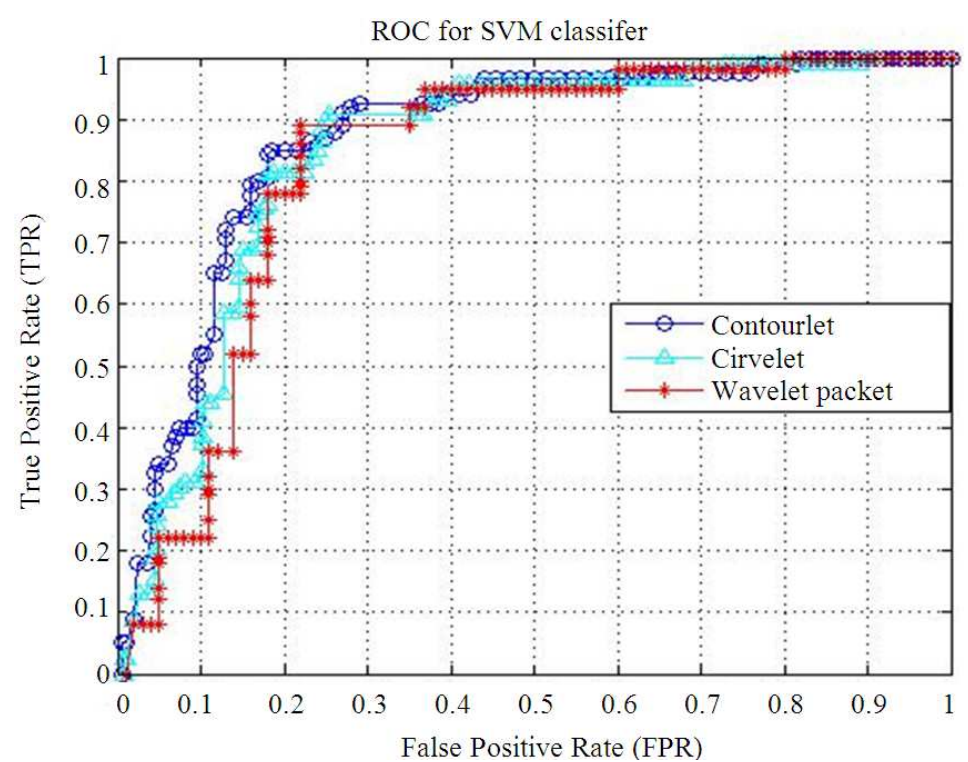

(a)

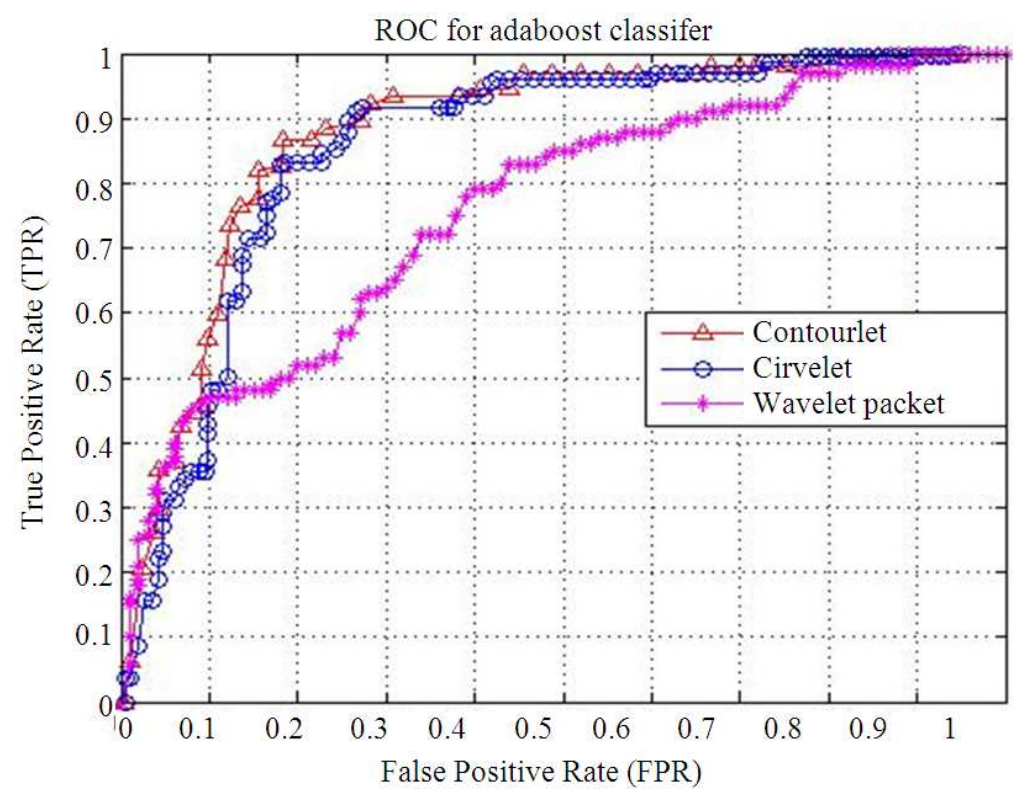

(b)

Fig. 5. (a) ROC curve for SVM classifier (b) ROC curve for adaboost classifier

\section{DISCUSSION}

Acharya et al. (2013a) developed a CAD system for classifying carotid plaques using 160 images. 32 Gray scale features were extracted and degree of stenosis was also obtained. SVM classifier with polynomial kernel order 1 was used to classify the plaques and classification accuracy of $90.66 \%$ was obtained. Acharya et al. (2012a) also proposed a CAD using gray scale features and classified the plaques as symptomatic or asymptomatic and classification accuracy of $82.4 \%$ was obtained. Kyriacou et al. (2009) used multilevel binary morphological analysis and achieved a classification accuracy of $73.7 \%$. Mougiakakou et al. (2007) realized a 
CAD system based on ultrasound image statistics, law's texture and neural network and achieved a high accuracy of $99.1 \%$. Classification accuracy of $73.1 \%$ was obtained by Christodoulou et al. (2003) using gray scale features and Fourier power spectrum. Classification accuracy of 93.1\% was got by Acharya et al. (2013b) using fuzzy classifier. All the above methods used different combination of gray scale features and classification methods. These methods either used more number of features or classification accuracies were less.

Discrete wavelet transform and averaging algorithm produced classification accuracy of $83.7 \%$ for SVM classifier (Acharya et al., 2012b). Tsiaparas et al. (2012) employed Wavelet transform, Ridgelet transform, curvelet transform and Wavelet packet and obtained highest overall classification accuracy of $79.2 \%$ for curvelet transform with SVM. Tsiaparas et al. (2011) obtained overall classification accuracy of $82.5 \%$ for Wavelet packet with SVM classifier. In the proposed method, we have employed contourlet transform and extracted energy, mean standard deviation and entropy features. SVM classifier with contourlet transform produced highest classification accuracy of $85.6 \%$. AUC for contourlet with SVM is 0.86. From the results, it is obvious that SVM classifier outperformed adaboost classifier in terms of classification accuracy. Even though higher accuracy of $91.7 \%$ was obtained by Acharya et al. (2011) using wavelet transform features and higher order spectra, this method used only smaller dataset. Hence more samples are to be used for validating the results.

\section{CONCLUSION}

In this study, a computer aided diagnostic system has been proposed by adapting the contourlet based texture features. The support vector machine classifier and adaboost classifier have been used to classify the plaques into symptomatic or asymptomatic. The performance of contourlet transform has been compared with Wavelet packet and curvelet transform features for both Adaboost and SVM. The contourlet features combined with SVM classifier has yielded better classification accuracy compared to other multi resolution texture features. ROC curve has also been obtained for both classifiers. AUC for contourlet based features with SVM is higher than that for other transform based features. By using the proposed $\mathrm{CAD}$ the objective decision can be taken by physician for better diagnosis of carotid diseases. This study used $2 \mathrm{D}$ ultrasound images for classification of carotid plaques. The drawback of this method is changes in thickness and volume of plaques cannot be measured. Hence 3 D ultrasound images can be employed to study the changes in plaque over time. The classification accuracy can also be improved further by using better combination of multiscale transform features and classification techniques.

\section{REFERENCES}

Acharya, U.R, O. Faust, V.S. Sree, A.P.C. Alvin and G. Krishnamurthi et al., 2011. Atheromatic ${ }^{\mathrm{TM}}$ : Symptomatic Vs. asymptomatic classification of carotid ultrasound plaque using a combination of HOS, DWT and texture. Proceedings of the Annual International Conference of the IEEE Engineering in Medicine and Biology Society, Aug. 30-Sept. 3, IEEE Xplore Press, Boston, MA., pp: 4489-4492. DOI: 10.1109/IEMBS.2011.6091113

Acharya, U.R, O. Faust, A.P.C. Alvin, S.V. Sree and F. Molinari et al., 2012a. Symptomatic Vs. asymptomatic plaque classification in carotid ultrasound. J. Med. Syst., 36: 361861-1871. DOI: 10.1007/s10916-010-9645-2

Acharya, U.R., O. Faust, S.V. Sree, F. Molinari and L. Saba et al., 2012b. An accurate and generalized approach to plaque characterization in 346 carotid US scans. IEEE Trans. Instrum. Measure., 61: 10451053. DOI: 10.1109/TIM.2011.2174897

Acharya, U.R., M.M.R. Krishnan, S.V. Sree, J. Sanches and S. Shafique et al., 2013a. Plaque tissue characterization and classification in ultrasound carotid scans: A paradigm for vascular feature amalgamation. IEEE Trans. Instrumentat. Measure., 62: 392-400. DOI: 10.1109/TIM.2012.2217651

Acharya, U.R., M.R. Mookiah, S.V. Sree, D. Afonso and J. Sanches et al., 2013b. Atherosclerotic plaque tissue characterization in 2D ultrasound longitudinal carotid scans for automated classification: A paradigm for stroke risk assessment. Med. Biol. Eng. Comput., 51: 513-523. DOI 10.1007/s11517012-1019-0

Bamberger, R.H. and M.J.T. Smith, 1992. A filter bank for the directional decomposition of images: Theory and design. IEEE Trans. Signal Process. 40: 882893. DOI: $10.1109 / 78.127960$

Burt, P.J and E.H. Adelson, 1983. The Laplacian pyramid as a compact image code. IEEE Trans. Commun., 31: 532-540. DOI: 10.1109/TCOM.1983.1095851 
Christodoulou, C.I., C.S. Pattichis, M. Pantziaris and A. Nicolaides, 2003. Texture-based classification of atherosclerotic carotid plaques. IEEE Trans. Med. Imag., 22 : 902-912. DOI: 10.1109/TMI.2003.815066

Do, M.N. and M. Vetterli, 2002. Contourlets: A directional multiresolution image representation. Proceedings of the IEEE International Conference on Image Processing, (CIP '02), IEEE Xplore Press, pp: 357-360. DOI: 10.1109/ICIP.2002.1038034

Do, M.N. and M. Vetterli, 2005. The contourlet transform: An efficient directional multiresolution image representation. IEEE Trans. Image Process., 14: 2091-2106. DOI: 10.1109/TIP.2005.859376

Katsigiannis, S., E.G. Keramidas and D. Maroulis, 2010. A contourlet transform feature extraction scheme for ultrasound thyroid texture classification. Eng. Intell. Syst.

Kyriacou, E., M.S. Pattichi, C.S. Pattichis A. Mavrommatis and C.I. Christodoulou et al., 2009. Classification of atherosclerotic carotid plaques using morphological analysis on ultrasound images. Applied Intell., 30: 3-23. DOI: 10.1007/s10489-0070072-0

Li, S. and J. Shawe-Taylor, 2004. Texture classification by combining wavelet and contourlet features. Proceedings of the Joint IAPR International Workshops on Structural and Syntactical Pattern Recognition and Statistical Pattern Recognition, Aug. 18-20, Springer Berlin Heidelberg, Lisbon, Portugal, pp: 1126-1134. DOI: $10.1007 / 978-3-540-27868-9 \_124$
Long, Z. and N.H. Younan, 2006. Contourlet spectral histogram for texture classification. Proceedings of the IEEE Southwest Symposium on Image Analysis and Interpretation, Mar. 26-28, Denver, CO., IEEE Xplore Press, pp: 31-35. DOI: 10.1109/SSIAI.2006.1633716

Moayedi. F., Z. Azimifar, R. Boostani and S. Kateb, 2010. Contourlet-based mammography mass classification using the SVM family. Comput. Biol. Med., 40: 373-383. PMID: 20181330. DOI: 10.1016/j.compbiomed.2009.12.006

Mougiakakou, S.G., S. Golemati, I. Gousias, A.N. Nicolaides and K.S. Nikita, 2007. Computer-aided diagnosis of carotid atherosclerosis Based on ultrasound image statistics, laws' texture and Neural networks. Ultrasound Med. Boil., 33: 26-36. DOI: 10.1016/j.ultrasmedbio.2006.07.032

Tsiaparas, N.N., S. Golemati, I. Andreadis, J. Stoitsis and I. Valavanis et al., 2012. Assessment of carotid atherosclerosis From b-mode ultrasound images using directional multiscale texture features. Measure. Sci. Technol., 23: 1-10. DOI: 10.1088/0957-0233/23/11/114004

Tsiaparas, N.N., S. Golemati, I. Andreadis, J.S. Stoitsis and I. Valavanis et al., 2011. Comparison of multiresolution features for texture classification of carotid atherosclerosis from B-mode ultrasound. IEEE Trans. Informat. Technol. Biomed., 15: 130137. DOI: $10.1109 /$ TITB.2010.2091511

WHO, 2013, The 10 leading causes of death in the world. World Health Organization. 